\title{
Mediterranean dietary intervention study of patients with rheumatoid arthritis
}

\section{Linda Hagfors}

Department of Food and Nutrition, Umeå University, Sweden

The overall aim of this thesis was to examine whether a modified Cretan Mediterranean diet can reduce signs and symptoms of rheumatoid arthritis (RA). This was investigated in a 3 month dietary intervention study in which 51 patients with well controlled, although active, RA of at least 2 years' duration took part. A further aim was to study the compliance with the experimental and control diets used in the study, and to validate the diet history interview method used to assess the dietary intake. The validation was carried out by means of biological markers of dietary intake.

From baseline to the end of the study the group that had adopted the Cretan Mediterranean diet (MD group; $n=26$ ) showed a significant improvement in nine out of 14 efficacy variables, while no changes was seen in the control diet group (CD group; $n=25$ ). Among the improvements observed in the MD group were a decrease in disease activity, improved physical function and improved vitality. Individually, 15 subjects in the MD group showed a moderate or better clinical improvement, based on the change in disease activity, compared with six subjects in the $\mathrm{CD}$ group.

According to the dietary assessments, the intake frequencies of antioxidant-rich food items increased in the MD group. This group also had a significantly higher intake of vitamin $\mathrm{E}$, vitamin $\mathrm{C}$ and selenium than the CD group. Despite the reported increase in the consumption frequencies of antioxidant-rich foods, the plasma levels of carotenoids, vitamin C, lipid-adjusted tocopherols, uric acid and urine malondialdehyde, a marker of oxidative stress, were unchanged at the end of the study. The plasma levels of retinol, vitamin $\mathrm{C}$ and uric acid were, however, correlated to variables related to disease activity.

Changes in the reported consumption of food groups with relevance to fat intake were also observed in the MD group. These changes included an increased intake of fish, shellfish and poultry, and a decreased intake of meat and high-fat dairy products. As a result, the total fat intake and the intake of saturated fatty acids were lower in the MD group than in the $C D$ group. In addition, the MD group had a lower ratio of n-6:n-3 fatty acids. A corresponding change in the relation between $n-6$ and $n-3$ fatty acids was also observed in s-phospholipids.

The validation of the diet history interview method showed that the diet history interview could capture the dietary intake fairly well. The validity of the reported dietary intake did not differ between the MD and the $\mathrm{CD}$ group, which indicates that the dietary assessment was not biased by the dietary intervention.

The thesis was conducted at the Department of Food and Nutrition, Umeå University, Umeå, Sweden. ISBN: 91-7305-497-6.

\section{Articles in the thesis}

1. Hagfors L, Leanderson P, Sköldstam L, Andersson J, Johansson G. Antioxidant intake, plasma antioxidants and oxidative stress in a randomized, controlled, parallel, Mediterranean dietary intervention study on patients with rheumatoid arthritis. Nutr J 2003; 2: 5.

2. Hagfors L, Nilsson I, Sköldstam L, Johansson G. Fat intake and composition of fatty acids in serum phospholipids in a randomized, controlled, Mediterranean dietary intervention study on patients with rheumatoid arthritis. Submitted for publication.

3. Hagfors L, Westerterp K, Sköldstam L, Johansson G. Validity of reported energy expenditure and reported intake of energy, protein, sodium and potassium in rheumatoid arthritis patients in a dietary intervention study. Eur J Clin Nutr 2005; 59 (2): 238-45.

4. Sköldstam L, Hagfors L, Johansson G. An experimental study of a Mediterranean diet intervention for patients with rheumatoid arthritis. Ann Rheum Dis 2003; 62: 208-14.

Linda Hagfors

E-mail: linda.hagfors@kost.umu.se 